\title{
Does donor cause of death affect the outcome of lung transplantation?
}

Anna Maria Ciccone, MDa

Ken C. Stewart, MDa

Bryan F. Meyers, MDa

Tracey J. Guthrie, RN, BSN ${ }^{a}$

Richard J. Battafarano, MDa

Elbert P. Trulock, MD

Joel D. Cooper, MDa

G. Alexander Patterson, MD

Background: Accumulating evidence suggests that the donor's cause of death may influence posttransplantation allograft function. We conducted a retrospective analysis of our adult lung transplant experience to investigate the influence of donor traumatic brain injury versus nontraumatic brain injury on posttransplantation outcome.

Methods: We retrospectively reviewed donor records and recipient medical charts for 500 consecutive lung transplants performed between July 1988 and December 1999. Recipient follow-up was complete, with a minimum follow-up of 1 year of survival.

Results: There were 295 and 205 donors in the traumatic and nontraumatic brain injury groups, respectively. Young male donors predominated in the traumatic brain injury group. Recipients receiving donor lungs from the traumatic and nontraumatic brain injury groups did not differ by age, sex, diagnosis, type of transplant (singlelung versus double-lung) or requirement for pretransplantation mechanical ventilatory assistance. Recipients did not differ in immediate or 24-hour $\mathrm{PaO}_{2} /$ inspired oxygen ratio, ventilation time, hospital stay, hospital mortality, or overall survival. Recipients of organs from donors who died of traumatic brain injury showed a higher severity and frequency of rejection episodes during the first year after transplantation. Freedoms from bronchiolitis obliterans syndrome at 5 years were $34.5 \%$ and $50.8 \%$ for recipients of organs from donors who died of traumatic and non-

From the Division of Cardiothoracic Surgery, Department of Surgery, ${ }^{\mathrm{a}}$ and Division of Pulmonary Medicine, Department of Internal Medicine, b Washington University School of Medicine, St Louis, Mo.

Read at the Eighty-first Annual Meeting of The American Association for Thoracic Surgery, San Diego, Calif, May 6-9, 2001.

Received for publication May 14, 2001; revisions requested July 10, 2001; revisions received Sept 5, 2001; accepted for publication Sept 13, 2001

Address for reprints: G. Alexander Patterson, MD, One Barnes-Jewish Plaza, 3108 Queeny Tower, St Louis, MO 63110 (E-mail: Pattersona@msnotes.wustl.edu).

J Thorac Cardiovasc Surg 2002;123:429-36

Copyright (C) 2002 by The American Association for Thoracic Surgery

0022-5223/2002 $\$ 35.00+0 \quad \mathbf{1 2 / 6 / 1 2 0 7 3 2}$

doi: $10.1067 / \mathrm{mtc} .2002 .120732$ traumatic brain injury, respectively $(P=.002)$.

Conclusions: The cause of donor brain death does not appear to influence early results of lung transplantation. Traumatic brain injury, or some phenomenon associated with it, may predispose a transplanted lung and its recipient toward more severe early rejection episodes and subsequent development of bronchiolitis obliterans syndrome.

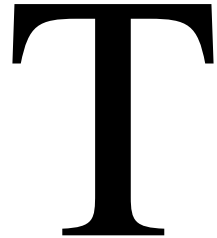

he insufficient supply of donor lungs results in prolonged waiting times and a substantial waiting list mortality among potential recipients. As a result of this donor shortage, lung transplant programs face intense pressure to use every possible donor lung. We have previously suggested that marginal donor lungs can be used without compromising early or late outcome. ${ }^{1,2}$ For the present, cadaveric donors after brain death remain the major source of lung grafts. The effect of brain death on pulmonary function is not clear, because knowledge of systemic changes occurring after this catastrophic event remains limited. Recent data in the organ transplant literature suggest that the donor's cause of death may be a factor in determining outcome after transplantation. 
TABLE 1. Patient characteristics

\begin{tabular}{|c|c|c|c|}
\hline & TBI $(n=295)$ & NTBI (n = 205) & $P$ value \\
\hline Donor age (y) & $23.9 \pm 9.3$ & $33.4 \pm 12.1$ & $<.001$ \\
\hline \multicolumn{4}{|l|}{ Donor sex (No.) } \\
\hline Female & $70(23.7 \%)$ & $108(52.7 \%)$ & \multirow[t]{2}{*}{.001} \\
\hline Male & $225(76.3 \%)$ & $97(47.3 \%)$ & \\
\hline \multicolumn{4}{|l|}{ Donor race (No.) } \\
\hline White & $236(80.0 \%)$ & $186(90.7 \%)$ & \multirow[t]{2}{*}{.001} \\
\hline Other & $59(20.0 \%)$ & $71(9.3 \%)$ & \\
\hline \multicolumn{4}{|l|}{ Donor CMV status (No.) } \\
\hline Negative & $167(56.6 \%)$ & $130(63.4 \%)$ & \multirow[t]{2}{*}{.13} \\
\hline Positive & $128(43.4 \%)$ & $75(36.6 \%)$ & \\
\hline Recipient age (y) & $46.6 \pm 11.6$ & $46.2 \pm 12.1$ & .71 \\
\hline \multicolumn{4}{|l|}{ Recipient sex (No.) } \\
\hline Female & $134(45.4 \%)$ & $113(55.1 \%)$ & \multirow[t]{2}{*}{.04} \\
\hline Male & $161(54.6 \%)$ & $92(44.9 \%)$ & \\
\hline \multicolumn{4}{|l|}{ Recipient diagnosis (No.) } \\
\hline Emphysema & $153(51.9 \%)$ & $114(55.4 \%)$ & \multirow[t]{5}{*}{.14} \\
\hline Pulmonary hypertension & $40(13.6 \%)$ & $16(7.8 \%)$ & \\
\hline Cystic fibrosis & $49(16.6 \%)$ & $30(14.7 \%)$ & \\
\hline Pulmonary fibrosis & $33(11.9 \%)$ & $22(10.8 \%)$ & \\
\hline Other & $20(6.8 \%)$ & $23(11.3 \%)$ & \\
\hline Recipient time waiting (d) & $515(178-599 *)$ & $395\left(235-695^{*}\right)$ & .001 \\
\hline \multicolumn{4}{|l|}{ Allograft type (No.) } \\
\hline Double-lung & $191(64.7 \%)$ & $144(70.2 \%)$ & \multirow[t]{3}{*}{.06} \\
\hline Single-lung & $103(34.9 \%)$ & $59(28.9 \%)$ & \\
\hline Heart-lung & $1(0.4 \%)$ & $2(0.9 \%)$ & \\
\hline \multicolumn{4}{|l|}{ Ischemic time (min) } \\
\hline Right lung & $290 \pm 76.6$ & $277.2 \pm 81.4$ & .1 \\
\hline Left lung & $313.9 \pm 91.7$ & $317.8 \pm 89.7$ & .67 \\
\hline Cardiopulmonary bypass & $71(24.1 \%)$ & $51(24.9 \%)$ & .83 \\
\hline
\end{tabular}

*Interquartile range (25\%-75\%).

In the renal transplant literature, short- and long-term function of transplanted kidneys from living donors is consistently superior to that seen with kidneys from cadaveric donors, which suggests that brain death may impact organ function. ${ }^{3-5}$ Shivalkar and colleagues ${ }^{6}$ demonstrated that a sudden rise in intracranial pressure resulted in poor functional recovery of experimental heart grafts, and in some cases this physiologic event was associated with irreversible myocardial damage. Takada and associates ${ }^{7}$ observed cytokine release and the activation of inflammatory cascades in an explosive brain death model and suggested that these events were responsible for changes in organ function. In contrast, a multicenter study showed that death from traumatic brain injury (TBI) had a positive influence on allograft survival in cardiac and renal transplantation. ${ }^{8}$

There is conflicting information regarding the impact of donor cause of death in the lung transplantation literature. Waller and colleagues ${ }^{9}$ did not demonstrate a difference in early graft function when the cause of donor death was classified as traumatic versus nontraumatic. On the other hand, reports have demonstrated that transplantation of lungs containing pulmonary embolic cerebral tissue after severe blunt or penetrating donor head trauma may be associated with increased recipient mortality. ${ }^{10,11}$

We performed this review to determine whether there was an association between the cause of donor brain death and clinical outcome after lung transplantation. We hypothesized that this central catastrophe could lead to lung injury and thus evoke a more intense host immunologic and inflammatory response, with a higher tendency toward rejection.

\section{Material and Methods \\ General}

We conducted a retrospective review of 500 adult patients receiving lung transplants at Barnes Jewish Hospital between July 1988 and December 1999. Background characteristics of the recipients are included in Table 1.

\section{Donor Data}

We recorded donor demographic characteristics, arterial blood gas analysis, $\mathrm{x}$-ray reports, and preremoval bronchoscopic results collected from United Network for Organ Sharing records. We classified the cause of donor brain death as TBI or nontraumatic brain injury (NTBI) according to cause of donor brain death recorded by United Network for Organ Sharing donor charts. Spontaneous intracranial hemorrhages and closed head injuries were the leading causes of death in the NTBI and TBI groups, respectively. Furthermore, we routinely categorized the donor groups into marginal or ideal groups according to previously published criteria. ${ }^{1}$

\section{Donor Operation}

The donor lung removal technique used by our team has been previously reported elsewhere. ${ }^{12}$ All 500 donors received broad-spectrum antibiotics intravenously within a few hours before organ retrieval. Donors were treated before organ removal with heparin $(4 \mathrm{mg} / \mathrm{kg}$ intravenously) and a bolus dose of prostaglandin $\mathrm{E}_{1}(500 \mu \mathrm{g})$ administered directly into the pulmonary artery immediately before aortic crossclamping. Lungs were flushed with antegrade cold modified Euro-Collins solution $(60-80 \mathrm{~mL} / \mathrm{kg})$. In the last 3 years we have administered pneumoplegia retrogradely into each pulmonary vein with the same solution $(5 \mathrm{~mL} / \mathrm{kg}) .{ }^{13} \mathrm{In}$ the last 2 years nitroprusside at $10 \mathrm{mg} / \mathrm{L}$ has been added to the flush solution. Lungs were harvested en bloc, preserved for transport by immersion in cold saline solution, and separated on return to our institution. Single-lung grafts destined for different centers were divided at the donor hospital.

\section{Recipient Data Collection}

We recorded preoperative and postoperative recipient data from recipient medical charts and our transplant database. Recipient follow-up was complete for all patients who received transplants through December 1999, with a minimum interval of 1 year for surviving patients.

\section{Recipient Criteria}

Our standard recipient selection criteria have been previously published elsewhere. ${ }^{14}$ Recipients are listed for transplantation when 
they have disabling lung disease with a limited prognosis and no other systemic illness that would complicate or be complicated by lung transplantation and immunosuppression. All patients listed for lung transplantation were enrolled in an active pulmonary rehabilitation program, with the exception of patients with pulmonary vascular disease.

Recipient Operative Technique and Postoperative Care We have described our operative techniques previously elsewhere. ${ }^{15,16}$ Early postoperative care was standardized. Mechanical ventilation was discontinued when gas exchange and weaning parameters permit. Routine empirical perioperative antibiotics with broad-spectrum gram-negative and gram-positive coverage was administered. Subsequent antibiotic selection was based on the results of donor and recipient bronchial cultures. Prophylaxis against Pneumocystis carinii consisted of trimethoprim-sulfamethoxazole given once daily until discharge, at which time the regimen was changed to 3 times per week. Recipients with cytomegalovirus (CMV) status mismatches carrying a high risk for infection (donor CMV seropositive and recipient CMV seronegative) received intravenous ganciclovir prophylaxis for 12 weeks. All patients were monitored for CMV viremia with weekly blood cultures.

A standard immunosuppressive protocol consisted of cyclosporine, corticosteroids, and azathioprine. We used other agents, including tacrolimus, mycophenolate mofetil, and sirolimus according to the clinical course. Immunosuppression induction typically included antithymocyte globulin. We performed routine flexible bronchoscopy, bronchoalveolar lavage, and transbronchial biopsies within 2 weeks and 3, 6, and 12 months, and on an asneeded basis thereafter.

A diagnosis of acute rejection required pathologic confirmation from a transbronchial biopsy specimen obtained during the first year after transplantation. Rejection was graded according to the grading system of the International Society for Heart-Lung Transplantation. ${ }^{17}$ Severe acute rejection was deemed to be present if any transbronchial biopsy specimen was graded A3 or higher. Increased frequency of rejection was ascribed to those patients with rejection scores of A2 or worse noted on 3 or more biopsy specimens during the first year. ${ }^{18}$ Bronchiolitis obliterans syndrome (BOS) was diagnosed and classified according to the grading system devised by the International Society of Heart and Lung Transplantation. ${ }^{19}$

\section{Statistical Analysis}

Normally distributed continuous data were expressed as mean \pm $\mathrm{SD}$. Interquartile ranges were used when continuous data were skewed. Categorical data were expressed as counts and proportions. Unrelated two-group comparisons were done with unpaired, 2-tailed $t$ tests for means of normally distributed variables and with Wilcoxon rank sum tests for skewed data. The $\chi^{2}$ or Fisher exact test was used to analyze differences among the categorical data. Kaplan-Meier estimates were used to depict survivals and freedoms from BOS. Survival and BOS-free survival comparisons between groups of patients were completed with the MantelHaenszel log-rank test.

Cox multivariate proportional hazards regression methods were used to discriminate risk factors for development of BOS
TABLE 2. Recipient outcome information

\begin{tabular}{lccc}
\hline & TBI (n= 295) & NTBI (n= 205) & $P$ value \\
\hline Acute & & & \\
$\quad \mathrm{PaO}_{2}$ /inspired oxygen ratio & & & \\
$4-6 \mathrm{~h}^{*}$ & $321 \pm 162.7$ & $328 \pm 166.6$ & .67 \\
$24 \mathrm{~h}^{*}$ & $210 \pm 111.3$ & $319 \pm 119.2$ & .37 \\
Mechanical ventilation (d) & $2(1-4 \dagger)$ & $2(1-4 \dagger)$ & .35 \\
Hospital stay (d) & $18(13-25 \dagger)$ & $18(13-25 \dagger)$ & .35 \\
Hospital mortality (No.) & $25(8.5 \%)$ & $13(6.3 \%)$ & .39 \\
Intermediate & & & \\
A3 rejection $\ddagger$ & $59(20.0 \%)$ & $24(11.7 \%)$ & .02 \\
A2 rejection $\times 3 \S$ & $49(16.6 \%)$ & $21(10.2 \%)$ & .02 \\
CMV & $71(24.1 \%)$ & $53(25.9 \%)$ & .67 \\
\hline
\end{tabular}

*Time after crossclamp release and reperfusion of allograft.

tInterquartile range $(25 \%-75 \%)$.

$\ddagger 0$ ne or more episodes of pathologically graded $\mathrm{A} 3$ rejection determined by transbronchial biopsy within first year after transplantation.

$\S$ Three or more episodes of pathologically graded $\mathrm{A} 2$ rejection or greater determined by transbronchial biopsy within first year after transplantation. One or more episodes of pathologically diagnosed CMV pneumonitis determined by transbronchial biopsy within first year after transplantation.

after transplantation. The time to death and the time to diagnosis of BOS were selected as the principal outcomes. All data analysis was performed with Systat (Systat 7.0 for Windows; SPSS Inc, Chicago, Ill). The regression models were constructed using dependent variables known or suspected to be independent predictors of the two outcomes based on previous published results and the univariate differences observed between our two exposure groups. The following categorical variables were considered: local versus distant donor, marginal versus ideal donor, donor death from TBI versus NTBI, donor sex, white versus nonwhite donor, recipient sex, recipient diagnosis (emphysema, cystic fibrosis, pulmonary hypertension, idiopathic fibrosis, other), single-lung versus double-lung transplant, recipient ventilator dependency at transplantation, donor CMV status, CMV mismatch stratified according to donor cause of death, presence of $\mathrm{A} 3$ rejection in recipient, 3 or more versus less than 3 distinct bouts of A2 rejection, and lineal quartile of recipient in overall experience (first, second, third, fourth).

The following continuous variables were considered: donor age in years, time from injury to declaration of brain death, time of declaration to procurement, recipient age in years, recipient days on the waiting list, and ischemic time in minutes.

\section{Results}

\section{Donor Characteristics}

Two hundred ninety-five donors died as a result of TBI and 205 died as a result of NTBI. Other donor characteristics are shown in Table 1. Our early experience appeared to use more TBI donors, but the difference in the distribution of the two groups was not significant $(P=.07)$. There was no difference between the TBI and NTBI cohorts with respect to recipient age, diagnosis, type of transplant (single-lung versus double- 


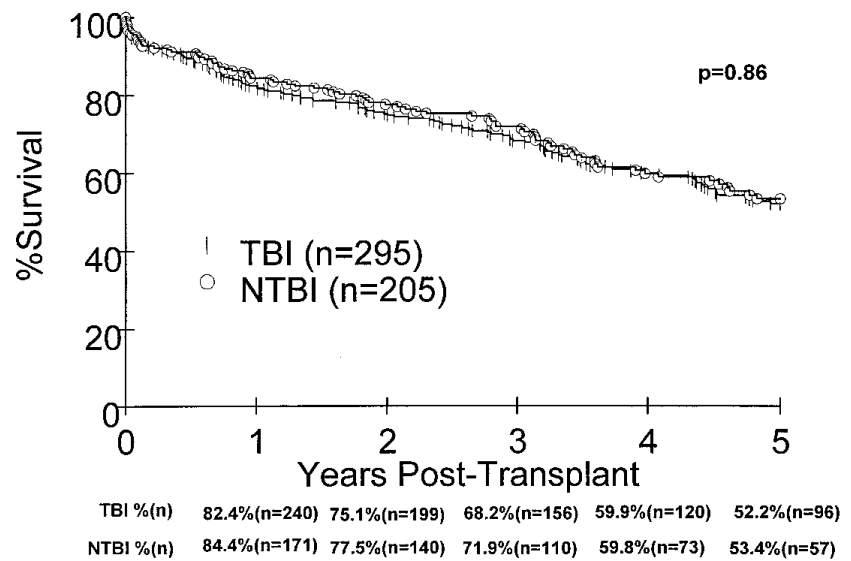

Figure 1. Kaplan-Meier survival plot of percentages of patients alive after transplantation stratified by donor cause of death as TBI (vertical lines) or NTBI (open circles). Percentages and numbers of patients available for analysis at each year are shown below graph.

lung versus heart-lung transplantation) or requirement for pretransplantation mechanical ventilatory assistance.

\section{Recipient Characteristics}

Pretransplantation waiting list time was significantly different between TBI and NTBI recipients. There was also a statistically significant difference in recipient sex, with a higher rate of male patients receiving lungs from TBI donors (Table 1).

\section{Recipient Operation}

Three hundred thirty-five patients underwent double-lung transplantation, 162 patients underwent single-lung transplantation, and 3 patients received heart-lung grafts. For TBI and NTBI recipients there was no statistically significant difference in the ischemic time, nor in the use of cardiopulmonary bypass during the transplantation procedure (Table 1).

\section{Postoperative Results}

Univariate analysis revealed comparable postoperative outcomes in the groups, with no significant difference in immediate or 24-hour $\mathrm{PAO}_{2}$ /inspired oxygen ratio, length of mechanical ventilation, length of hospitalization, or hospital mortality (Table 2). However, TBI recipients showed a higher severity and frequency of rejection episodes in the first year after transplantation (Table 2). Five-year survivals were $52.2 \%$ and $53.4 \%$ for TBI and NTBI recipients, respectively $(P<.8$; Figure 1$)$.

Kaplan-Meier estimates of freedom of BOS at 5 years were $34.5 \%$ and $50.8 \%$ for TBI and NTBI recipients, respec-

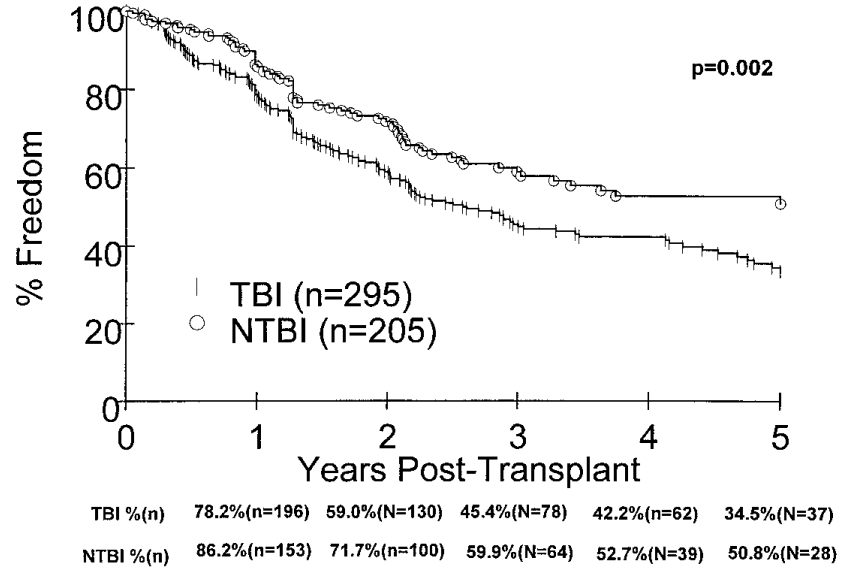

Figure 2. Kaplan-Meier survival plot of percentages of patients free from BOS after transplantation stratified by donor cause of death as TBI (vertical lines) or NTBI (open circles). Percentages and numbers of patients available for analysis at each year are shown below graph.

tively $(P=.002$; Figure 2$)$. Cox proportional hazards analysis of risk factors for death did not result in any independent risk factors, and the overall model lacked statistical significance. The results of the Cox multivariate analysis of risk factors for the development of BOS after transplantation are shown in Table 3. TBI and the occurrence of A3 rejection in the first year after transplantation were statistically significant risk factors for development of BOS. Recent timing of transplantation was a negative risk factor for BOS.

\section{Discussion}

Cadaveric donors after brain death will remain the major source of lung grafts for the foreseeable future. Brain death can be associated with injury to organs intended for transplantation. ${ }^{3,4}$ In fact, the irreversible loss of brain function will probably lead to a progressive deterioration in function of all organs. Interest has recently focused on the physiologic changes occurring during brain death, although the mechanism of the subsequent systemic changes remains unclear. ${ }^{5}$ Explosive brain death results in significant hemodynamic alteration and upward regulation of proinflammatory mediators and cell-surface molecules in donor organs. Experimental studies have demonstrated that a sudden increase in intracranial pressure leads to a series of pathophysiologic changes that have been described as "autonomic storm" and are characterized by catecholamine release and histopathologic changes in potentially transplantable organs..$^{20,21}$ Hemodynamic changes include increased systemic vascular resistance and increased mean arterial pressure, with acute elevation of left atrial pressure. Pulmonary capillary integrity may be disrupted, resulting in 
acute pulmonary edema. Endocrine changes in epinephrine, cortisol, and thyroxine levels increase organ damage as aerobic metabolism is progressively inhibited. Brain injury enhances the effects of cytokines and other alloantigenindependent factors that play a role in subsequent allograft chronic rejection, such as humoral activity and mononuclear cell infiltration. ${ }^{22}$

Despite the numerous differences between TBI and NTBI donors, our data do not support the hypothesis that donor cause of death affects recipient survival. Furthermore, we could not detect an immediate difference between these groups of recipients in their physiologic outcomes after transplantation. However, TBI recipients did appear to have a higher severity and frequency of acute rejection episodes within 1 year after transplantation. The risk of development of BOS was higher among our TBI recipients than among our NTBI recipients. The increased risk for BOS among TBI recipients appeared to be independent of the additional risk caused by severe acute rejection.

Various factors have been associated with increased risk for subsequent BOS, including CMV infection, frequency and severity of early lung allograft rejection episodes, biopsy findings of lymphocytic bronchiolitis, increased donor age, increased ischemic time, the development in the recipients of donor-specific anti-HLA antibodies during postoperative course, and type of transplant (single-lung vs double-lung transplant). ${ }^{18,23}$ We evaluated many of these risk factors by including them in our Cox model assessing risk factors for BOS. The inclusion of donor age, recipient age, ischemic time, frequency of acute rejection, and transplant type as independent variables did not affect the relative risk for BOS attributable to TBI in our data.

There are a number of limitations to this retrospective review. First, we acknowledge the possibility that TBI is associated with BOS without causing it. For this to be true there must be some other factor closely associated with both TBI and BOS. We reported a higher number of male patients in the TBI donor group and a higher number of male patients receiving TBI grafts. Presumably, this inequity is due to the fact that TBI donors are more often male because males more often are affected by trauma. Male recipients are more likely to receive male donor lungs because of the size issues, and therefore male recipients, male donors, and TBI donors are linked more than chance would dictate. We do not think that donor or recipient sex can have a role in our finding of TBI as associated with BOS, however, because no previous reports have suggested that donor or recipient sex is a risk factor for BOS.

Novick and coworkers ${ }^{24}$ have suggested that older donor age is associated with increased risk for BOS. With respect to the donor age, we found that TBI donors were younger than were NTBI donors; however, this group still had an increased incidence of subsequent BOS.
TABLE 3. Multivariate Cox proportional hazards model of risk factors for BOS after transplantation

\begin{tabular}{lrccc}
\hline Variable & No. & Relative risk & 95\% CI & Pvalue \\
\hline A3 rejection episode* & 83 & 2.115 & $1.550-2.886$ & $<.001$ \\
Donor TBI & 295 & 1.432 & $1.078-1.900$ & .013 \\
Time of transplantation & & & & \\
$\quad$ 1st quartile (reference) & 125 & 1 & - & - \\
2nd quartile & 125 & 0.836 & $0.609-1.149$ & .27 \\
3rd quartile & 125 & 0.553 & $0.374-0.816$ & .003 \\
4th quartile & 125 & 0.272 & $0.160-0.462$ & $<.001$ \\
Primary diagnosis & & & & \\
$\quad$ Emphysema (reference) & 267 & 1 & - & - \\
Pulmonary fibrosis & 55 & 0.916 & $0.599-1.401$ & .687 \\
Cystic fibrosis & 79 & 1.307 & $0.914-1.869$ & .142 \\
Pulmonary hypertension & 56 & 1.197 & $0.780-1.837$ & .411 \\
Other & 43 & 0.85 & $0.491-1.470$ & .56 \\
\hline
\end{tabular}

${ }^{*}$ One or more episodes of pathologically graded A3 rejection determined by transbronchial biopsy within first year after transplantation.

There may be other covariates that were unmeasured and perhaps unevenly distributed between our TBI and NTBI groups. TBI donors are usually given a higher number of blood transfusions or blood products which could actually render the graft at a higher level of antigenicity. The evaluation of the TBI donor is complicated by the fact that the resuscitation and stabilization of such donors are less controlled. The TBI donors may have a higher incidence of aspiration of gastric contents at the time of injury which in turn may produce subsequent gram-negative infections. Circumstances of endotracheal intubation may differ, as TBI donors are more likely to be intubated at the scene of the accident, potentially in a less sterile environment. Donor hypotension and donor lung contusion could be other factors expected to cause tissue damage and could thus predispose the TBI lungs to an increased risk of BOS. Because these factors are unmeasured potential causes of lung injury that are likely to be unequally distributed between the TBI and NTBI groups, they remain as potential confounders in this analysis.

There is also a possibility that TBI is involved in a causal pathway that leads to BOS. In fact, previous work in other organs have demonstrated end-organ damage as a result of TBI and have emphasized that different causes of brain death may produce different somatic effects. This same mechanism may lead to pulmonary damage and set in motion a chain of events leading to an escalated host response to the graft lungs.

Furthermore, we have to consider that the differences seen here were due to chance alone. We have arbitrarily divided a group of 500 patients into groups on the basis of a single exposure criterion and then compared the exposure 
groups on the basis of numerous (20) criteria. By chance alone we would expect them to differ in at least 1 or 2 criteria at a level of significance of $P<.05$.

The possibility also exists that donors or recipients have been misclassified according to exposure status (TBI vs NTBI) or outcome status (BOS or death). The exposure status was straightforward and not subjective, because donor cause of death was provided by the United Network for Organ Sharing from records created at the time of transplant. The end point of death is also unequivocal, but that of BOS is less certain. It is certainly possible that the most recent cohort of transplant recipients have been underdiagnosed for BOS simply because of the periodic, scheduled nature of the surveillance bronchoscopies. Because TBI donors were more prevalent during the early experience, such an oversight in recent recipients would lead to overestimation of the BOS risk caused by TBI. Although this is possible, the inclusion of the quartile variable in the Cox regression model should account for differences in the BOS risk in early versus late recipients.

We are also reluctant to accept that TBI is associated with a higher risk for BOS while not being associated with a higher risk for death. The plausibility of the association between TBI and BOS is weakened by the reported finding that BOS remains the leading cause of late complications and mortality after lung transplantation. The patient sample size is large and the effect size of BOS risk is moderate, but the consistency that must be seen to accept the association is lacking. We therefore accept our findings as an exploratory observation in need of confirmation.

\section{Conclusions}

Our study demonstrated equivalent early outcomes and no significant difference in long-term survival between TBI and NTBI groups. However, TBI was associated with severity of acute rejection and development of BOS. Whether recipients whose donors have died of TBI need a closer surveillance or earlier intensification of immunosuppression therapy warrants verification with further studies to provide the clinical relevance of this fascinating finding.

\section{References}

1. Sundaresan S, Semenkovich J, Ochoa L, Richardson G, Trulock EP, Cooper JD, et al. Successful outcome of lung transplantation is not compromised by the use of marginal donor lungs. J Thorac Cardiovasc Surg. 1995; 109:1075-80.

2. Meyers BF, Lynch JP, Trulock EP, Guthrie TJ, Cooper JD, Patterson GA. Lung transplantation: a decade of experience. Ann Surg. 1999;230:362-71.

3. Terasaki PI, Cecka JM, Gjertson DW, Takemoto S. High survival rates of kidney transplants from spousal and living-related donors. $N$ Engl J Med. 1995;333:333-6.

4. Pratschke J, Wilhelm MJ, Kusaka M, Hancock WW, Tilney NL. Acute rejection of rat renal allografts is accelerated by donor brain death. Transplant Proc. 1999:31:874-5.
5. Novitzky D. Detrimental effects of brain death on the potential organ donor. Transplant Proc. 1997;29:3770-2.

6. Shivalkar B, Van Loon J, Wieland W, Tjandra-Maga TB, Borgers M, Plets $\mathrm{C}$, et al. Variable effects of explosive or gradual increase of intracranial pressure on myocardial structure and function. Circulation. 1993;87:230-9.

7. Takada M, Nadeau KC, Hancock WW, Mackenzie HS, Shaw GD, Waaga AM, et al. Effects of explosive brain death on cytokine activation of peripheral organs in the rat. Transplantation. 1998;65:1533-42.

8. Busson M, N'Doye P, Benoit G, Hannoun L, Adam R, Pavie A, et al. Donor factors influencing organ transplant prognosis. Transplant Proc. 1995;27:1662-4.

9. Waller DA, Thompson AM, Wrightson WN, Gould FK, Corris PA, Hilton CJ, et al. Does the mode of donor death influence the early outcome of lung transplantation? A review of lung transplantation from donors involved in major trauma. J Heart Lung Transplant. 1995; 14:318-21.

10. Rosendale BE, Keenan RJ, Duncan SR, Hardesty RL, Armitage JA, Griffith BP, et al. Donor cerebral emboli as a cause of acute graft dysfunction in lung transplantation. $J$ Heart Lung Transplant. 1992:11:72-6.

11. Simonetti VA, Basha MA, Allenspach L, Klosterman KG, Nakhleh R, Higgins RS. Donor cerebral tissue pulmonary emboli in a functioning transplanted lung. Clin Genet. 1998;12:504-7.

12. Sundaresan S, Trachiotis GD, Aoe M, Patterson GA, Cooper JD. Donor lung procurement: assessment and operative technique. Ann Thorac Surg. 1993;56:1409-13.

13. Varela A, Cordoba M, Serrano-Fiz S, Burgos R, Montero CG, Tellez $\mathrm{G}$, et al. Early lung allograft function after retrograde and antegrade preservation. J Thorac Cardiovasc Surg. 1997;114:1119-20.

14. Trulock EP. Lung transplantation. Am J Respir Crit Care Med. 1997; 155:789-818.

15. Meyers BF, Patterson GA. Technical aspects of adult lung transplantation. Semin Thorac Cardiovasc Surg. 1998;10:213-20.

16. Meyers BF, Sundaresan RS, Guthrie T, Cooper JD, Patterson GA. Bilateral sequential lung transplantation without sternal division eliminates posttransplantation sternal complications. J Thorac Cardiovasc Surg. 1999;117:358-64.

17. Yousem SA, Berry GJ, Cagle PT, Chamberlain D, Husain AN, Hruban $\mathrm{RH}$, et al. Revision of the 1990 working formulation for the classification of pulmonary allograft rejection: Lung Rejection Study Group. J Heart Lung Transplant. 1996;15:1-15.

18. Bando K, Paradis IL, Similo S, Konishi H, Komatsu K, Zullo TG, et al. Obliterative bronchiolitis after lung and heart-lung transplantation. J Thorac Cardiovasc Surg. 1995;110:4-14.

19. Cooper JD, Billingham M, Egan T. A working formulation for the standardization of nomenclature and for clinical staging of chronic dysfunction in lung allografts. J Heart Lung Transplant. 1993;12:7136.

20. Novitzky D, Wicomb WN, Cooper DK, Rose AG, Fraser RC, Barnard $\mathrm{CN}$. Electrocardiographic hemodynamic and endocrine changes occurring during experimental brain death in the chacma baboon. Heart Transplant. 1984;4:63-9.

21. Shanlin RJ, Sole MJ, Rahimifar M, Tator CH, Factor SM. Increased intracranial pressure elicits hypertension, increased sympathetic activity, electrocardiographic abnormalities and myocardial damage in rats. J Am Coll Cardiol. 1988;12:727-36.

22. Tullius SG, Tiney NL. Both alloantigen-dependent and -independent factors influence chronic allograft rejection. Transplantation. 1995;59:313-8.

23. Smith MA, Sundaresan S, Mohanakumar T, Trulock EP, Lynch JP, Phelan DL, et al. Effect of development of antibodies to HLA and cytomegalovirus mismatch on lung transplantation survival and development of bronchiolitis obliterans syndrome. $J$ Thorac Cardiovasc Surg. 1998;116:812-20.

24. Novick RJ, Bennett LE, Meyer DM, Hosenpud JD. Influence of graft ischemic time and donor age on survival after lung transplantation. $J$ Heart Lung Transplant. 1999;18:425-31. 


\section{Discussion}

Dr John V. Conte (Baltimore, $M d$ ). This is an interesting article that looked at the largest number of donors and the impact of traumatic and nontraumatic injuries on outcomes to date. The results have the advantage, unlike other articles, of long-term follow-up, which I think adds a lot. Dr Ciccone, you mentioned that in your TBI group the largest percentage of donors died of blunt head injury. Did you have the opportunity perhaps to look at those who had projectile or open-head injury types as opposed to the blunt head injury? Might that have had any impact on the outcomes? A second and related question is whether you have had the opportunity to look at the time from injury until brain death and see whether that had any impact on outcomes. You hypothesized in your article that part of the injury was caused by the explosive release of inflammatory mediators, and perhaps it should be the impact of the time that the donor was exposed to these mediators, as opposed to the mechanism of release of these mediators, that might have the effect.

On another note, you appropriately did a Cox analysis and pointed out some of the factors that have been associated with BOS. There have been other factors through the years that have been associated, less convincingly than the ones you looked at, and I was wondering whether you looked at such factors as reperfusion injury and the incidence of diffuse alveolar disease among the lung biopsy specimens that you retrieved to see whether there was any correlation there.

Also, did you go back and do retrospective crossmatch or retrospective HLA analyses of the donors and recipients, particularly the HLA-DR locus, to see whether there was any impact on those variables? And did you look at all types of infection, not just CMV infection? That was the only infectious agent that you mentioned in the article.

Finally, you mentioned that it might be a good idea to look at this group of patients a little bit more closely. Have you changed your surveillance bronchoscopy schedule on these patients, and have you changed your immunosuppressive management of these patients as a result of the findings in this study?

Dr Ciccone. Dr Conte, please repeat your first question.

Dr Conte. Did you look at the different types of TBI, closedhead injury versus open-head injury, for example, from a gunshot wound?

Dr Ciccone. We did not subcategorize types of TBI. We divided the time from injury to harvest and we divided from injury to declaration of death and declaration of death to harvest. Of course, the time from injury to declaration of death was shorter in the TBI group than in the NTBI group, and this was the time where we found a statistical difference. There was no difference in the time from declaration of death to lung removal.

We included in our regression analysis 30 separate variables (including ischemic reperfusion injury and diffuse alveolar damage) that we did not report to avoid overloading the article with too many data. None of these resulted in a statistically significant difference. This was also true for CMV status. We did not look at the HLA matching.

Your last question concerns our current treatment program. At this time we have not changed surveillance or immunosuppression of patients receiving lungs from donors who died of TBI, because we are unsure of the clinical relevance of this fascinating finding. However, we think that this finding warrants verification with other programs' experience and may provide insight into the factors leading to BOS. In this regard, all of us know that any information is helpful.

Dr Malcolm M. DeCamp, Jr (Cleveland, Ohio). I continue to be fascinated by the whole mechanism of brain death and early posttransplantation outcome, as our heart transplant experience from the Cleveland Clinic found just the opposite, that hearts from donors who died of trauma had better survival than did those from donors who died of nontraumatic causes. So this is clearly an area that needs to be further investigated.

I had one question regarding CMV status. I noticed that there was no difference in CMV status between the TBI and NTBI donors. How about the CMV mismatch issue of positive donor organs into seronegative recipients, which has been implicated as leading to problems with chronic rejection?

Finally, could you just comment on your routine immunosuppressive regimen? Induction regimens have been shown to decrease the risk of early acute rejection or at least severe acute rejection, though they have not shown a survival advantage. It would appear that you saw a little bit more grade III or severe rejection in this TBI group. Thus this might be an ideal group to consider varying immunosuppression on the basis of the mechanism of brain death of the donor.

Dr Ciccone. The analysis that we presented documents donor CMV status only. As you point out, there was no difference in CMV status between TBI and NTBI donors. We have not yet completed the analysis of donor-recipient CMV mismatch for these groups. Recipients with CMV status mismatches that carry high risk for infection (donor CMV positivity and recipient CMV negativity) receive intravenous ganciclovir prophylaxis for 12 weeks. All patients are monitored for CMV viremia with weekly blood cultures. I did not understand your last question about the severity of the acute rejection.

Dr DeCamp. You reported an increased incidence of severe rejection in the TBI group than in the NTBI group, although it did not appear to make a difference in terms of survival. Induction therapy, for what it's worth, has been shown to decrease both the incidence and the severity of acute rejection, although these differences have not translated into a survival advantage. Would you consider the use of induction immunotherapy for recipients of organs from donors who died of TBI?

Dr Ciccone. As I previously stated, we have not changed any of our treatment at the moment. Our belief is that these patients are already heavily immunosuppressed with our current regimen. Our standard immunosuppressive protocol consists of cyclosporine, corticosteroids, and azathioprine. Other agents, including tacrolimus, mycophenolate mofetil and sirolimus, are used as second line drugs depending upon clinical course. Historically we have used antithymocyte globulin as induction therapy. However, in recent months we have been using basiliximab in cases of CMV mismatch with seronegative recipients.

Dr Axel Haverich (Hannover, Germany). We are yet left with no answer as to the BOS syndrome in the TBI subgroup. At least in Europe most of those who have TBI have also a lot of injuries in other places. They are often operated on for peripheral bleeding 
or for abdominal bleeding. Have you looked into the number of blood transfusions or blood products, which are usually not given in cases of NTBI death? Blood products could actually place the graft at a higher level of antigenicity and therefore would be able to explain both the higher incidence of acute rejection and also BOS. So have you looked at the number of blood transfusions in the two groups?

Dr Ciccone. Dr Haverich, you raise a potentially important question. When we began this review, we attempted to examine the influence of donor blood transfusion on outcome. However, this analysis was impossible because the data were missing for many donors in our early experience.

Dr Michael F. McGrath (Norfolk, Va). Obviously donors come from a number of different kinds of hospitals and settings. Were you able to split out those patients who had early neurosurgical intervention to decompress the rapid rise in intracranial pressure and see whether that had any effect on the donor lungs?

Dr Ciccone. No, we did not include this in our analysis.

Access to The Journal of Thoracic and Cardiovascular Surgery Online is reserved for print subscribers!

Full-text access to The Journal of Thoracic and Cardiovascular Surgery Online is available for all print subscribers. To activate your individual online subscription, please visit The Journal of Thoracic and Cardiovascular Surgery Online, point your browser to http://www.mosby.com/jtcvs, follow the prompts to activate your online access, and follow the instructions. To activate your account, you will need your subscriber account number, which you can find on your mailing label (note: the number of digits in your subscriber account number varies from 6 to 10). See the example below in which the subscriber account number has been circled:

\section{Sample mailing label}

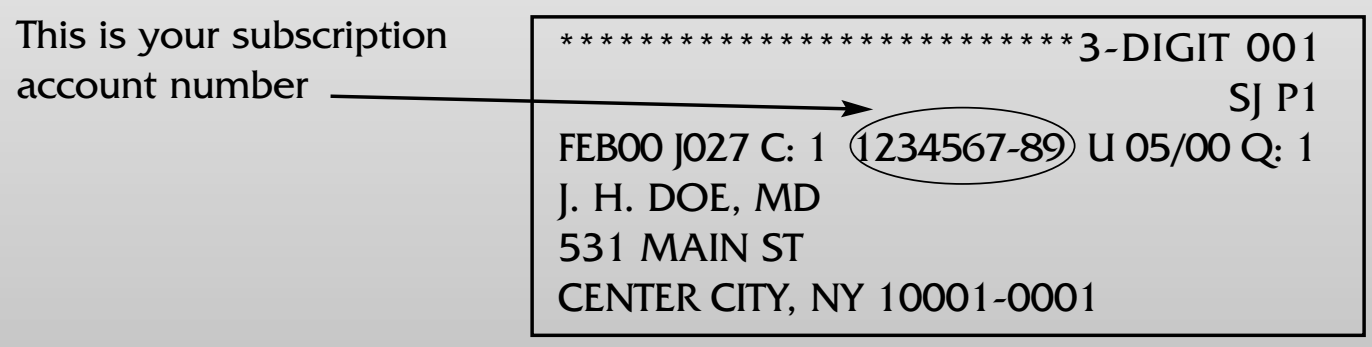

Personal subscriptions to The Journal of Thoracic and Cardiovascular Surgery Online are for individual use only and may not be transferred. Use of The Journal of Thoracic and Cardiovascular Surgery Online is subject to agreement to the terms and conditions as indicated online. 